\title{
Recent Developments In Monolithic Phase-Locked Semiconductor Laser Arrays
}

Katz, J, Kapon, E, Margalit, S, Yariv, A.

J Katz, E Kapon, S Margalit, A. Yariv, "Recent Developments In Monolithic Phase-Locked Semiconductor Laser Arrays," Proc. SPIE 0465, Spatial Light Modulators and Applications I, (13 August 1984); doi: 10.1117/12.941426 
Recent developments in monolithic phase-locked semiconductor laser arrays

J. Katz

Jet Propulsion Laboratory, 4800 Oak Grove Drive, Pasadena, California 91109

E. Kapon, S. Margalit, A. Yariv

California Institute of Technology, Pasadena, California 91109

\section{Abstract}

Coherent combination of the power of several semiconductor lasers fabricated on the same substrate has been the subject of an intense research effort in recent years, the main motivation being to obtain higher power levels than those available from a single laser in a stable radiation pattern. Best results reported so far include 2.6 Watts cw emitted power and less than $1^{\circ}$ far-field angle (in the array plane) in arrays where all the lasers are electrically connected in parallel. A different type of coherent array, where each element has a separate contact, has been recently demonstrated. While requiring the more complex two-level metallization technology, applying a separate contact to each laser provides an additional degree of freedom in the design and the operation of monolithic arrays. The separate contacts can be employed to tailor the near-field and far-field distributions and to compensate for device-to-device nonuniformities. Furthermore, the control of the currents of the array elements allows the performance of a variety of other functions, such as beam scanning, spectral mode control, wavelength tuning and control of the mutual coherence between array elements.

\section{Introduction}

Semiconductor injection lasers have many properties that make them the preferred choice for light sources in many electro-optical applications. However, their advantages of small size and weight, long lifetime, high power efficiency and the possibility to directly modulate them at high rates are offset by the fact that the power levels emitted by a single device into a stable radiation pattern $(\leq 50 \mathrm{mw})$ are too low for some applications. One possible method for overcoming this limitation is by a monolithically implementation of coherent power combining of several semiconductor lasers. There are two basic features of coherent (vs. incoherent) power combining. First, when the power of the lasers is combined incoherently, each laser emits light in its own individual spectrum, resulting in a potentially wider overall spectral envelope. In coherent power combining, all the array elements have the same spectrum. Secondly, phase-locking of the lasers causes a reduction in the far-field beam divergence angle, in a manner similar to a phased-array of antennas. Coherent power combining itself can be implemented by different methods, including external cavity, monolithic and hybrid configurations.

\section{Conventional semiconductor laser arrays}

In this paper, the subject of linear (one-dimensional) monolithic phase-1ocked laser arrays will be discussed. To date, most of the published works on the subject of semiconductor laser coherent power combining have reported various configurations of laser arrays on common substrates. ${ }^{1,2}$ A schematic cross section of such a generic array is depicted in Figure 1. The lasers are placed in a close proximity to each other so there is sufficient interaction to assure their phase-locking, either by evanescent field coupling, leaky wave coupling or other mechanisms. In addition, smaller separations between array elements reduce the number of grating lobes in the far-field of the array, and this is a desirable feature since, in most applications, a single-lobed fundamental mode operation is preferred. Another possible solution--which has not been implemented yet--to the grating lobes problem is the randomization of the location of the individual lasers around the periodic array structure. ${ }^{3}$ In most devices demonstrated so far, the center to center spacing of the lasers is about $10 \mu \mathrm{m}$ for gain-guided lasers and $5 \mu \mathrm{m}$ for index guided lasers, where the shorter distance is probably required because of the smaller coupling in that case. Semiconductor laser arrays have been demonstrated by several research groups. Array configurations included gain-guided stripe lasers, leaky buried-heterostructure lasers, and others. Best results reported include 2.6 Watts $\mathrm{cw}$ of emitted power ${ }^{4}$ and less than $1^{\circ}$ of far-field angle in the array plane. 5 (It should be noted, however, that these results were not reported under the same operating conditions.) An important feature of the arrays of the type shown in Figure 1 is that a single contact is applied to all the lasers, i.e., they are internally connected electrically in parallel. This has important consequences regarding the operation of the array, since individual diode elements cannot be accessed independently and, thus, there is no control over the internal functioning of the device. For example, it is 
frequently found that, due to non-uniformities that exist among the individual lasers, not al1 the array elements reach the lasing threshold at a certain (total) current level.

\section{Separate Contact Semiconductor Laser Arrays}

In response to this problem we have recently designed, fabricated and tested a novel type of 8-element phase-locked semiconductor laser array where each diode laser has its own separate contact. ${ }^{6}$ The schematic configuration of the array is shown in Figure 2 . The array elements are stripe geometry lasers with approximately 4-5 $\mu$ m stripe width. Adjacent lasers are isolated with proton implantation. Center to center spacing between lasers is $9 \mu \mathrm{m}$, and their threshold currents are typically $60 \mathrm{~mA}$. While requiring the more complex two-level metallization technology, applying a separate contact to each laser provides an additional degree of freedom in the design of monolithic arrays, which can be used, for example, to compensate for device-to-device non-uniformities, tailor the near-field and farfield distributions and perform other functions. This array can also serve as a small-scale "laboratory" for investigating the basic physical processes governing the coupling interactions among the integrated lasers.

Experimental results include the demonstration of control over the near-field (Figure 3$)^{6}$ and the far-field (Figure 4$)^{6,7}$ distributions of several elements of the array. Effects of non-uniformities among the lasers is clearly seen in Figure $3 \mathrm{e}$, where the array elements are shorted together and fed through a single current source. This near-field pattern should s è contrasted against the one depicted in Figure $3 a$, which shows the near-fields of the same lasers with each one fed through its separate contact. Figure 4 shows that by adjusting the currents through the lasers, they can lock either out of phase (Figure 4b) or in-phase (Figure 4c), as clearly deduced from the respective far-field patterns. Wavelength tuning was achieved by varying the gain in lasers which operate below threshold and which are in close proximity to lasing elements (Figure 5) ${ }^{8}$. An interesting feature is that the number of longitudinal modes in the phase-locked array can be made much smaller than that of each individual laser operating by itself. Even single longitudinal mode operation has been achieved, which is unusual for gain-guided lasers. In addition, basic beam scanning has been demonstrated, and coupling mechanisms were investigated and found to be different in gain-guided lasers as compared to the evanescent wave coupling typically assumed for real index-guided lasers. The amount and strength of coupling between any two elements of the array can also be controlled by varying the currents of the diodes between them (Figure 6 ). ${ }^{9}$

\section{Conclusions}

In conclusion, phase locked semiconductor laser arrays may become very useful devices in many electro-optical applications involving coherent power combining for higher power levels, beam shaping, and wavelength tuning and control. Realization of most of these functions is made possible by providing each laser in the array with its own separate contact so that it can be independently addressed.

\section{Acknowledgements}

The research described in this paper was performed jointly by the Jet Propulsion Laboratory and the Applied Physics Department, California Institute of Technology, under contracts with the National Aeronautics and Space Administration, the Office of Naval Research and the National Science Foundation. E. Kapon would like to acknowledge the support of the Weizmann post-doctoral fellowship.

\section{References}

1. Scifres, D. R., Burnham, R. D., and Streifer, W., "Phase-Locked Semiconductor Laser Array," App1. Phys. Lett., Vo1. 33, pp. 1015-1017. 1978.

2. Katz, J., Margalit, S., and Yariv, A., "Diffraction-Coupled Phase-Locked Semiconductor Laser Array," App1. Phys. Lett., Vo1. 42, pp. 554-556, and references within. 1983. 3. Katz, J., "Power Combining of Semiconductor Injection Lasers: a Review," TDA Progress Report, Vol. 42-70, pp. 87-94, Jet Propulsion Laboratory, Pasadena, California. 1982. 4. Streifer, W., Burnham, R. D., Paoli, T. L., and Scifres, D. R., "Phase-Locked (GaAl)As Diode-Laser Arrays," Opt. Soc. Am. Annual Meeting, Oct.17-20, 1983, New Orleans, Louisiana (paper WA5).

5. Scifres, D. R., Streifer, W., and Burnham, R. D., "Experimental and Analytic Studies of Coupled Multiple Stripe Diode Lasers," IEEE J. Quantum Electron., Vo1. QE-15, pp. 917-922. 1979 .

6. Katz, J., Kapon, E., Lindsey, C., Margalit, S., Shreter, U., and Yariv, A., "Phase Locked Semiconductor Laser Array with Separate Contacts," App1. Phys. Lett., Vo1. 43, pp. 521-523. 1983 .

7. Katz, J., Kapon, E., Lindsey, D., Margalit, S., and Yariv, A., "Far-Field Distributions of Semiconductor Phase-Locked Arrays with Multiple'Contacts," Electron. Lett., Vo1. 19, pp. 660-662. 1983 . 
8. Kapon, E., Katz, J., Margalit, S., and Yariv, A., "Longitudinal Mode Control in Integrated Semiconductor Laser Phase-Arrays by Phase Velocity Matching," to be published in App1. Phys. Lett., Vo1. 44, Jan. 15, 1984.

9. Kapon, E., Katz, J., Lindsey, C., Margalit, S., and Yariv, A., "Control of Mutual Phase Locking of Monolithicaliy Integrated'Semiconductor'Lasers," App1. Phys. Lett., Vol. 43, pp. 421-423. 1983 .

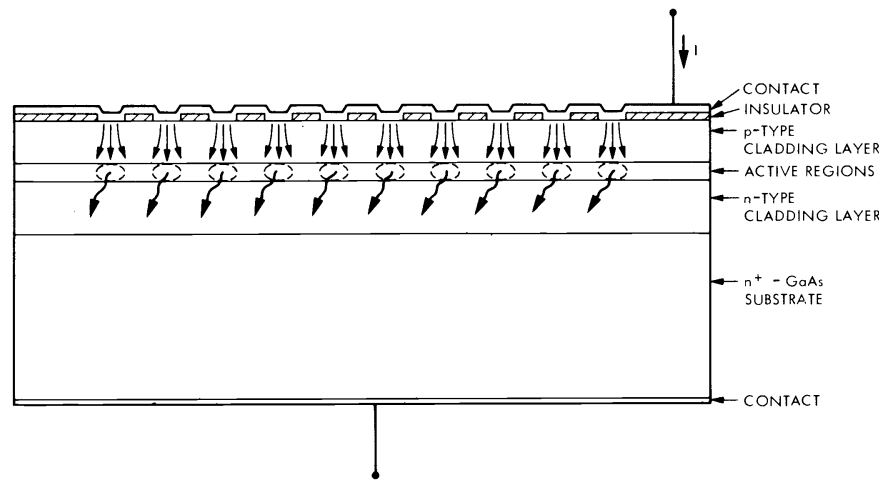

Figure 1. Schematic cross section of a monolithic phase-1ocked semiconductor laser array.

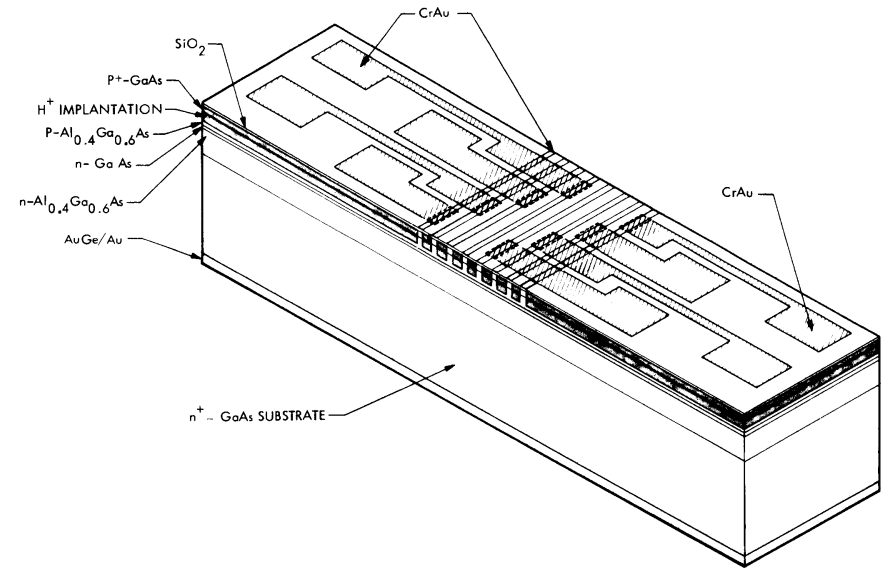

Figure 2. Schematic structure of the separate contact array. 


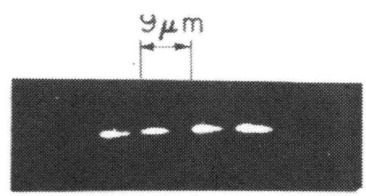

(a)

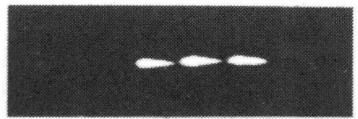

(b)

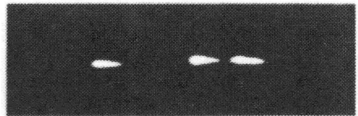

(c)

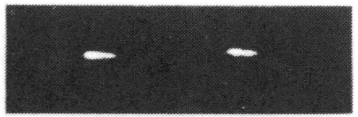

(d)

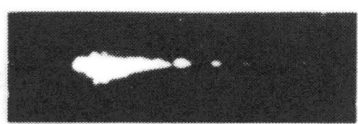

(e)

Figure 3. Near-field patterns of a group of four elements of the separate contact array:

(a) a11 four lasers operating;

(b,c) three lasers operating;

(d) two lasers operating;

(e) al1 four lasers connected in paralle1 and fed through one current.

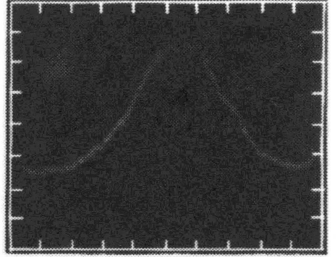

(a)

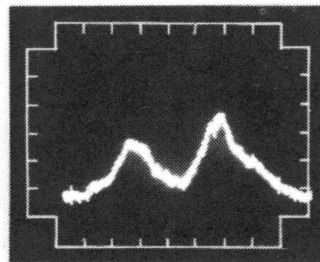

(b)

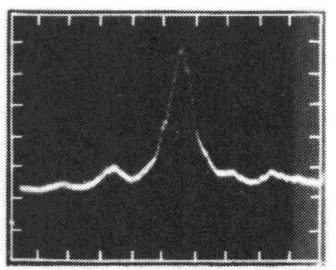

(c)

Figure 4 .

(a) Far-field pattern of a single element of the array.

(b) Far-field of a 4-element separate contact array with lasers operating in anti-phase mode.

(c) Far-field of a 4-element separate contact array with lasers operating in in-phase mode.

Horizontal scale: 2 degrees/div. Vertical scale: arbitrary units 


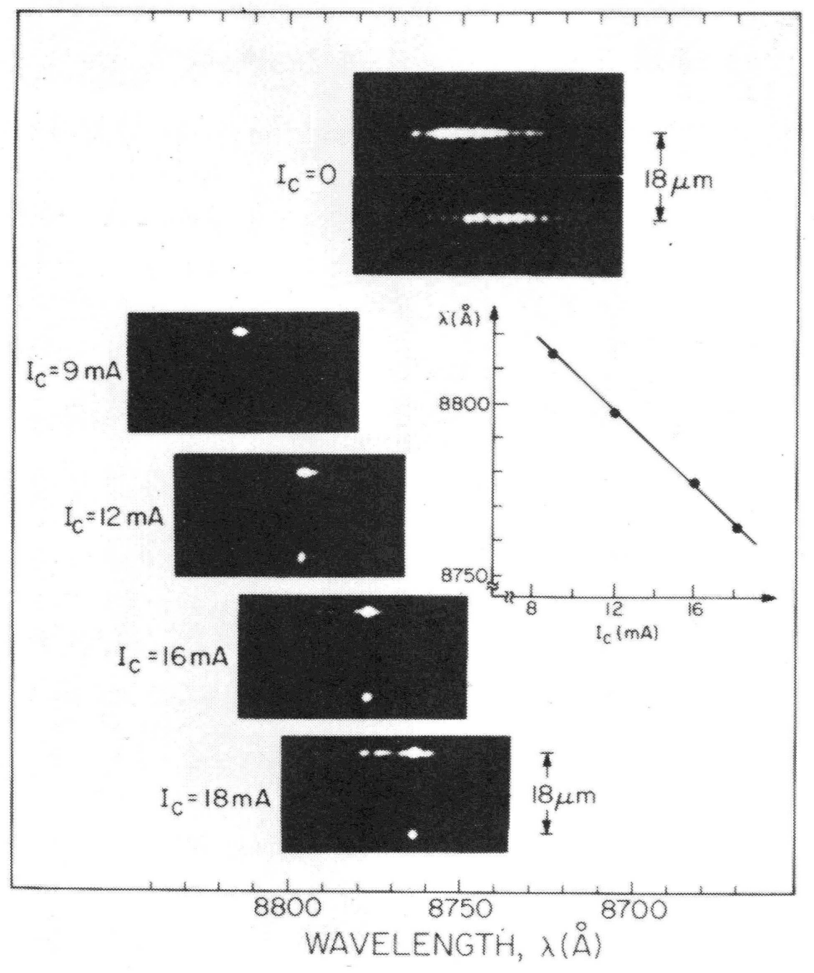

Figure 5. Spectrally resolved near-fields of an array of two lasers, separated by $18 \mu \mathrm{m}$, for various values of the current $I_{C}$ through the stripe between them. The insert shows the wavelength $\lambda$ of the main longitudinal mode for different currents $I_{C}$.
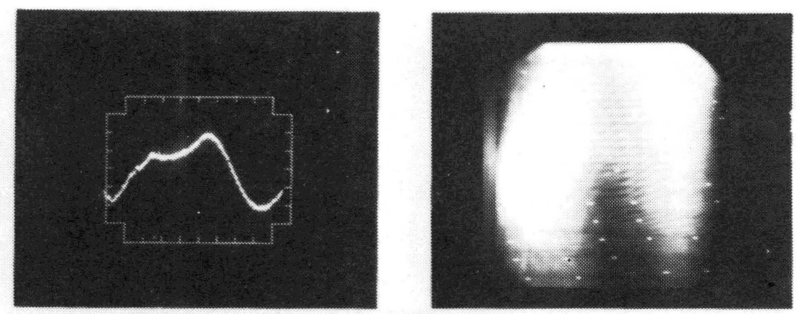

(a)
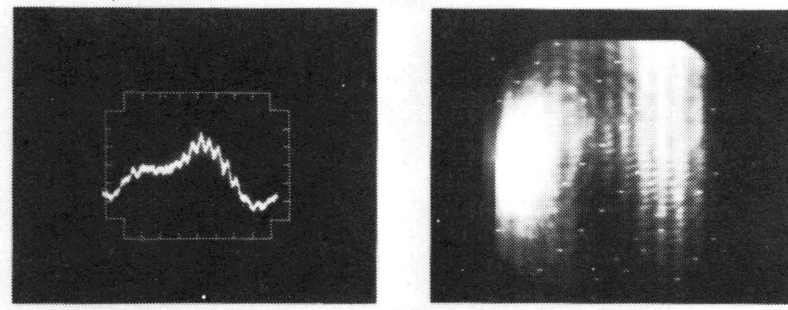

(b)
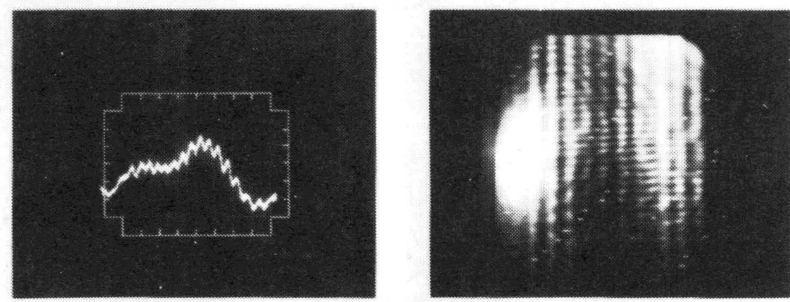

(c)

Figure 6. Far-field scans and photographs for two lasers separated by $45 \mu \mathrm{m}$ :

(a) with no biased lasers between them,

(b) with one biased laser at $\mathrm{I}=0.9 \mathrm{I}_{\mathrm{th}}$,

(c) with yet another laser biased at $I=0.1 \mathrm{Ith}$. The horizontal divisions in the far-field scans are at $2.5^{\circ}$ separation. 